\title{
The Expression of Wnt5a Gene Throughout Definitive Endoderm Induction Process in Induced Pluripotent Stem Cells
}

\author{
Shahrokh Lorzadeh ${ }^{1,2}$, Negar Azarpira (iD ${ }^{3,}$, , Saeid Ghavami ${ }^{4,5,6}$ and Leila Kohan ${ }^{7}$ \\ ${ }^{1}$ Department of Genetics, Fars Science and Research Branch, Islamic Azad University, Marvdasht, Iran \\ ${ }^{2}$ Department of Genetics, Marvdasht Branch, Islamic Azad University, Marvdasht, Iran \\ ${ }^{3}$ Transplant Research Center, Shiraz University of Medical Sciences, Shiraz, Iran \\ ${ }^{4}$ Department of Human Anatomy and Cell Science, Rady Faculty of Health Sciences, Max Rady College of Medicine, University of Manitoba, Winnipeg, Canada \\ ${ }^{5}$ Faculty of Medicine, Katowice School of Technology, Katowise, Poland \\ ${ }^{6}$ Autophagy Research Center, Shiraz University of Medical Sciences, Shiraz, Iran \\ ${ }^{7}$ Department of Biology, Arsanjan Branch, Islamic Azad University, Arsanjan, Iran \\ "Corresponding author: Transplant Research Center, Shiraz University of Medical Sciences, Shiraz, Iran. Email: negarazarpira@yahoo.com
}

Received 2020 October 20; Revised 2020 October 26; Accepted 2020 October 28.

\begin{abstract}
Background: Induced pluripotent stem cells (iPSCs) have the ability to proliferate indefinitely and differentiate into three germ layers of ectoderm, mesoderm, and endoderm. Definitive induction is the first and the most delicate stage of differentiation of various iPSC-derived organs. It has been found that the Wnt signaling pathway implicates in embryogenesis, organogenesis, and cell communication.

Objectives: In the present study, we aimed to investigate the expression pattern of the Wnt5a gene as an indicator of non-canonical Wnt signaling activity during definitive endoderm induction of iPSCs.

Methods: Human iPSCs (RSCB0042) were acquired from Royan stem cell bank of Royan Institute (Tehran, Iran). The iPSCs were cultured on a feeder layer of mitomycin-inactivated mouse embryonic fibroblasts (MEF), and iPSC colonies were collected for embryoid body (EB) generation by suspension culture method. Then endoderm induction step was performed using a series of small molecules. The quantitative real-time PCR was used to assess the mRNA expression of wnt5a, Nanog, OCT4, SOX17, and FOXA2 genes.

Results: The production of efficient EBs confirmed by a decrease in Nanog and Oct4 gene expression and the success of DE (definite endoderm) induction step was confirmed by a high expression level of DE specific genes, Sox17, and FoxA2. A significant upregulation of Wnt5a in EB samples and a minor decrease at day 4 was observed. However, the differentiation process followed by an incremental fashion in Wnt5a mRNA expression starting from day 4 of differentiation among the samples of days 6 and 8 (DE stage).

Conclusions: Our results suggest that Wnt5a is more activated at the later steps of endoderm induction rather than the early steps, which may be due to the stimulation of canonical Wnt signaling. Finding the expression level of Wnt5a could rise insights for developing more efficient differentiation induction protocols.
\end{abstract}

Keywords: Non-Canonical Wnt Signaling, Differentiation, Definitive Endoderm, Induced Pluripotent Stem Cells, Gene Expression

\section{Background}

The reprogramming techniques of somatic cells by the introduction of pluripotency transcription factors led to the generation of induced pluripotent stem cells (iPSCs). Like embryonic stem cells, iPSCs have the ability to proliferate indefinitely and differentiate into three germ layers of ectoderm, mesoderm, and endoderm (1). Definitive endoderm cells can be differentiated into internal organs such as lungs, liver, pancreas, and thyroid (2). Therefore, definitive induction is the first and the most delicate stage of differentiation of various iPSC-derived organs. There are diverse signaling pathways involved in definitive endoderm generation, including TGF $\beta, \mathrm{SHH}$, and Wnt pathways $(3,4)$.
Wnt signaling pathway comprises two different classes of canonical and non-canonical Wnt pathways. Both are controlled by a set of glycoprotein ligands, which are recognized by their cognate cell surface receptors, Frizzled family, and coreceptors of low-density-lipoprotein-related protein5/6 (LRP5/6). The attachment of Wnt ligands to frizzled receptors leads to the activation of Dishevelled (Dvl) protein $(5,6)$. In canonical Wnt signaling, $\beta$-catenin plays the central role, which is under constant degradation by a destructive complex, consist of, glycogen synthase kinase-3 $\beta$ (GSK-3 $\beta$ ), adenomatous polyposis coli (APC), casein-kinase $1 \alpha(\mathrm{CK} 1 \alpha)$, and Axin $(6,7)$. By induction of canonical Wnt signaling by specific ligands such as Wnt3a, $\beta$-catenin is 
translocated into the nucleus and activates Tcf/Lef transcription factors to initiate the expression of target genes $(8,9)$. Non-canonical Wnt/planar cell polarity (PCP) signaling is induced by different sets of ligands, such as Wnt4 and Wnt5a $(10,11)$. The signal transduction cascade in Wnt/PCP pathway is initiated independent of $\beta$-catenin, from Dvl to secondary effectors, c-Jun N-terminal kinase (JNK), and the small GTPases of Rho and Rac, which lead to cytoskeleton rearrangement and activation of AP1 transcription factors to alter gene expression (12).

Wnt signaling pathway has been established to be implicated in embryogenesis, organogenesis, and cell communication. However, canonical Wnt signaling is responsible for cell hemostasis and proliferation whereas, Wnt/PCP is in favor of differentiation of stem cells into various types of cell lineages such as adipocytes, osteocytes, and cardiac cells $(6,13-17)$.

\section{Objectives}

This study was designed to investigate the expression pattern of the Wnt5a gene as an indicator of non-canonical Wnt signaling activity during definitive endoderm induction of iPSCs.

\section{Methods}

\subsection{Cell Culture}

The commercially available Human iPSCs (RSCB0042) were purchased from Royan stem cell bank (Royan Institute, Tehran, Iran). Briefly, iPSCs were cultured on a feeder layer of mitomycin (M7949, Sigma, Germany) inactivated mouse embryonic fibroblasts (MEF) incubated at $37^{\circ} \mathrm{C}, 5 \% \mathrm{CO}_{2}$ and humidity of 80 - 90\% (18). Dulbecco modified eagle medium (DMEM)/Ham's F12 (Gibco, Life Technologies, USA) was used as the basic medium for iPSC culture supplemented with $20 \%$ knockout serum replacement (KOSR) (10828020, Gibco), $5 \mu \mathrm{g} / \mathrm{mL}$ selenium, insulintransferrin-selenium (ITS) (I1884, Gibco), 100 Unit/mL penicillin, $100 \mu \mathrm{g} / \mathrm{mL}$ streptomycin (P4333, Sigma-Aldrich), 0.1 mM non-essential amino acids (M7145, Sigma-Aldrich), 0.1 mM 2-mercaptoethanol (15433, Merck) and $12 \mathrm{ng} / \mathrm{mL}$ basic fibroblast growth factor (bFGF) (F0291, Sigma-Aldrich). The culture media was exchanged every other day, until iPSC colonies covered at least 70\% of the culture flask surface. Then iPSC colonies were collected for embryoid body (EB) generation by suspension culture method (19). Subsequently, iPSC colonies were detached from culture flask by cell scraper and resuspended in ultra-low attachment plates (SPL, Korea) with DMEM/F12 medium supplemented with 20\% fetal bovine serum (FBS) (Gibco), $100 \mathrm{Unit} / \mathrm{mL}$ penicillin, and $100 \mu \mathrm{g} / \mathrm{mL}$ streptomycin for three days. Then, EBs were collected and cultured in six-well cell culture plates (SPL) coated with $2 \%$ gelatin (G1890, Sigma, Germany) and kept for 3 days at $37^{\circ} \mathrm{C}, 5 \% \mathrm{CO}_{2}$, and humidity of $80-90 \%$. The endoderm induction step was performed using a series of small molecules based on our previous researches (4). Therefore, the previous medium is removed and EBs were cultured in RPMI1640 medium (21875034, Gibco) containing $30 \mathrm{ng} / \mathrm{mL}$ of Activin A(H4666, Sigma) and $3 \mu \mathrm{M}$ CHIR99021 (SML1046, Sigma) without serum supplement. Further CHIR99021 was removed from the medium after 24 hours with a gradual increase in serum supplement in the medium. Therefore, the next day, the medium was replaced with a fresh one containing $0.2 \% \mathrm{KOSR}$ and $30 \mathrm{ng} / \mathrm{mL}$ of Activin A. After 48 hours, the medium was removed with the replacement of $2 \%$ KOSR containing medium, which was continued for three days. Cell samples were harvested for RNA extraction at days 0 (iPSC), 3 (EB), 4, 5, 6, and 8 (definitive endoderm).

\subsection{Quantitative Real-time PCR}

Collected cell samples were washed by Phosphate buffered saline (PBS), and then total RNA was extracted by RnaSol mRNA extraction kit (Alphabio, USA) according to the manufacturer's instruction. In order to prepare complementary DNA (cDNA), PrimeScript First Strand cDNA Synthesis Kit (6110A, Takara, Japan) was used with $500 \mathrm{ng}$ of total RNA. The quantitative real-time PCR was performed by StepOnePlus instrument (Applied Biosystem, USA) and SYBR@ Premix EX TaqTM II kit (RR820A, Takara, Japan). The unique forward and reverse primers for Wnt5a mRNA (sequences and melting temperatures are presented in Table 1) were uniquely designed by AlleleID software (version 7.5). Amplification was performed in $10 \mu \mathrm{L}$ aliquots with $10 \mathrm{pM}$ of each primer. GAPDH gene was used as an internal control for expression normalization. All reactions were done in triplicate.

\subsection{Statistical Analysis}

Real-time data were analyzed by LinRegPCR software (version 2017.1) to identify the highly efficient reactions (95\%105\% and 0.99). Statistical analysis and fold changes were calculated by GenEX software (v.7.0.2.164). The Fold changes ratio was quantified against iPSC. One-way ANOVA was used for the comparison between cell samples with Tukey-Kramer's for post hoc test. The P-value of less than 0.05 was considered statistically significant. 


\begin{tabular}{|c|c|c|c|}
\hline Gene Name & Sequence (5' to 3') & Tm & Product Length (BP) \\
\hline \multirow{2}{*}{ Wnt family member $5 A$ (WNT5A) } & F-GGATGGCTGGAAGTGCAATG & 59.54 & \multirow{2}{*}{112} \\
\hline & R-TTCATACCTAGCGACCACCA & 58.14 & \\
\hline \multirow{2}{*}{ GAPDH } & F-GGACTCATGACCACAGTCCA & \multirow{2}{*}{60} & \multirow{2}{*}{119} \\
\hline & R-CCAGTAGAGGCAGGGATGAT & & \\
\hline
\end{tabular}

\section{Results}

\subsection{Differentiation Induction}

The success of the differentiation process was verified by the assessment of specific marker genes. Initially, the pluripotency characteristics of iPSCs were confirmed by the observation of pluripotency marker genes, Nanog, and Oct4 genes expression. Also, EBs were efficiently produced from fully grown iPSC colonies and showed a decrease in Nanog and Oct4 gene expression ( $0.175 \pm 0.043 ; \mathrm{P}<0.001$ and $0.213 \pm 00.053 ; \mathrm{P}<0.001$ respectively). The significant downregulation of Nanog and Oct4 genes during the rest of the differentiation induction was the indicator of successful escape from pluripotency (Figure 1A). The success of DE induction step was confirmed by the assessment of DE specific genes, including Sox17 and FoxA2. Both genes showed elevated expression levels compared to iPSC (9.937 $\pm 0.464 ; \mathrm{P}<0.001$ and $209.615 \pm 1.08 ; \mathrm{P}<0.001$ respectively) (Figure 1B).

\subsection{Wnt5a Expression}

As shown in Figure 2, the expression profile of Wnt5a mRNA represents non-canonical Wnt signaling during the differentiation induction process. We observed a significant upregulation of Wnt5a in EB samples (5.033 \pm 1.55 ; P $<0.001)$. However, a minor decrease was observed on day 4 (3.715 $\pm 0.279 ; \mathrm{P}<0.001)$. The differentiation process followed by an incremental fashion in Wnt5a mRNA expression started from day 4 of differentiation ( $4.508 \pm 0.314 ; \mathrm{P}<$ 0.001) among the samples of days 6 and 8 (DE stage) (6.9751 $\pm 1.216 ; \mathrm{P}<0.001$ and $7.709 \pm 0.926 \mathrm{P}<0.001)$.

\section{Discussion}

The evolutionary conserved non-canonical Wnt signaling is involved in development, cell proliferation, and differentiation in coordination with canonical Wnt signaling $(12,20)$. Wnt/PCP is a type of non-canonical Wnt signaling, which is activated by the Wnt5a ligand is a well-known antagonist of the canonical pathway (10, 21-23). Canonical Wnt signaling is mostly involved in cell proliferation and stemness maintenance and only the early stages of differentiation induction $(13,23)$. Therefore, Wnt/PCP should be activated after the initial stage of endoderm induction. In this study, we demonstrate the expression of the Wnt5a gene is decreased at the early endoderm induction stage, which is consistent with the introduction of canonical Wnt signaling inducer, namely chir99021. However, during the rest of the endoderm induction process, Wnt5a expression ramped up. Therefore, the specific stage of Wnt5a expression, as non-canonical Wnt signaling, has a decisive role during definitive endoderm induction and differentiation toward different lineages such as the pancreas (3). Baksh et al. have shown that Wnt5a can suppress the canonical Wnt signaling pathway, which can promote the osteogenic differentiation of MSCs (20) since canonical Wnt signaling functions only at the early differentiation induction as suggested by Paige et al. (24). In the same way, Vijayaragavan et al. reported that the non-canonical Wnt signaling is required for the embryonic stem cells to escape from pluripotency (25). Moreover, Wnt5a was shown to promote the cardiac cells, dental papilla cells, chondrocyte, adipocyte, and osteogenic differentiation (15-17, 26, 27). Therefore, it can be postulated that Wnt/PCP is activated at the later steps of endoderm differentiation and positively promotes the differentiation process. The study of Wnt5a expression may provide new insights into developing more effective differentiation protocols considering the fact that definitive endoderm induction is the initial step for the generation of many internal organs and is the most delicate step throughout the differentiation induction.

\subsection{Conclusion}

Non-canonical Wnt signaling contributes to developmental pathways and differentiation. We studied the expression profile of the Wnt5a gene, during endoderm induction and suggest that Wnt5a is more activated at the later steps of endoderm induction rather than the early step, which may be due to the stimulation of canonical Wnt signaling. Understanding the expression pattern of Wnt5a may provide insights into developing more efficient differentiation induction protocols. 
A

$\underline{\text { Oct4 }}$

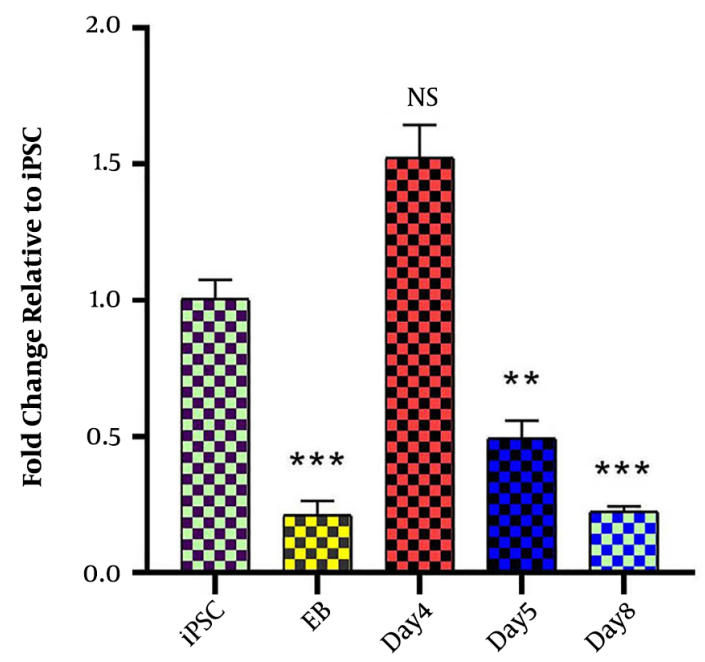

Differentiation Stages

C

FoxA2

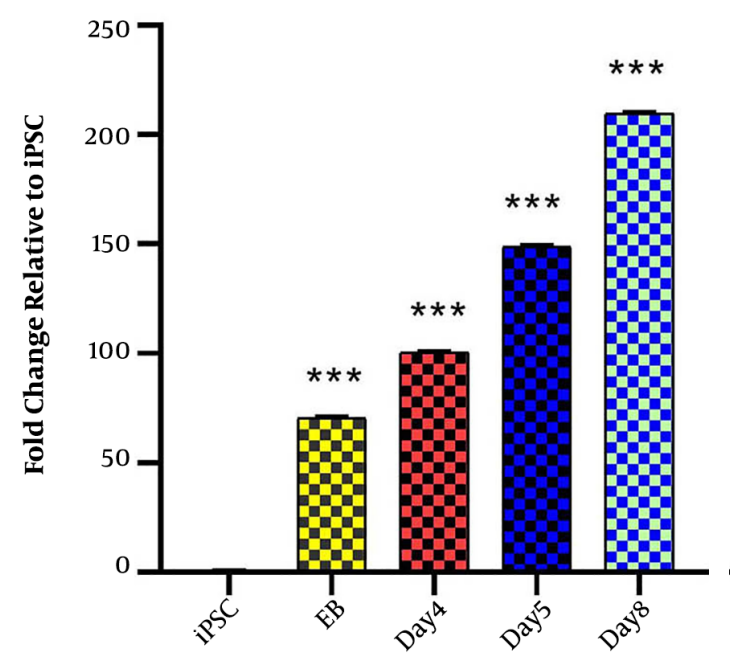

Differentiation Stages
B

Nanog

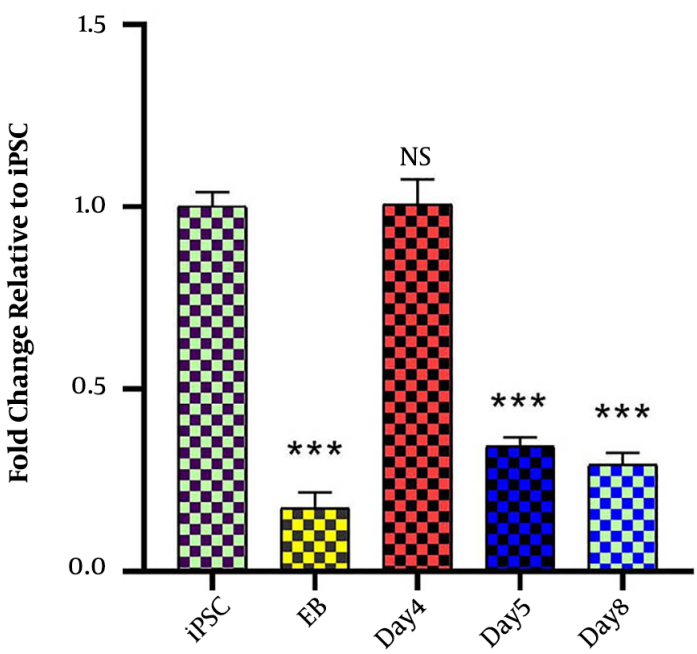

Differentiation Stages

D

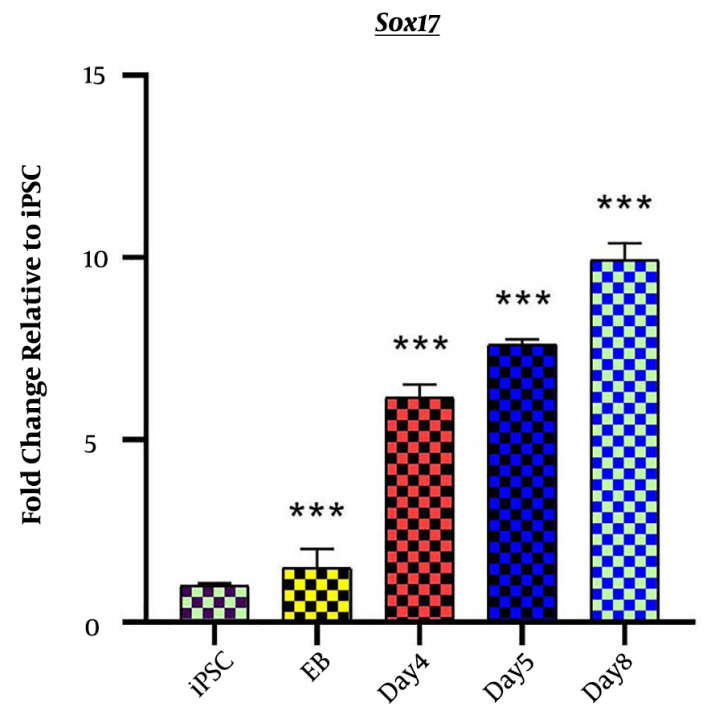

Differentiation Stages

Figure 1. Stage-specific differentiation marker genes; Fold change ratio of mRNA expression is presented on "Y" axis, while differentiation stages, including iPSCs, Embryoid body (EB), day 4 of differentiation (day 4), day 5 of differentiation (day 5), and endoderm (day 8) are depicted on "X" axis. (A) The expression profile of pluripotency-specific genes, Oct4 and Nanog, represents the successful scape form stemness at day 8 (endoderm stage). (B) The expression profile of endoderm marker genes, Sox17 and FoxA2, represents the completion of endoderm induction. ${ }^{*} \mathrm{P}<0.05 ;{ }^{* *} \mathrm{P}<0.01 ;{ }^{* * *} \mathrm{P}<0.001$; NS: not-significant. 


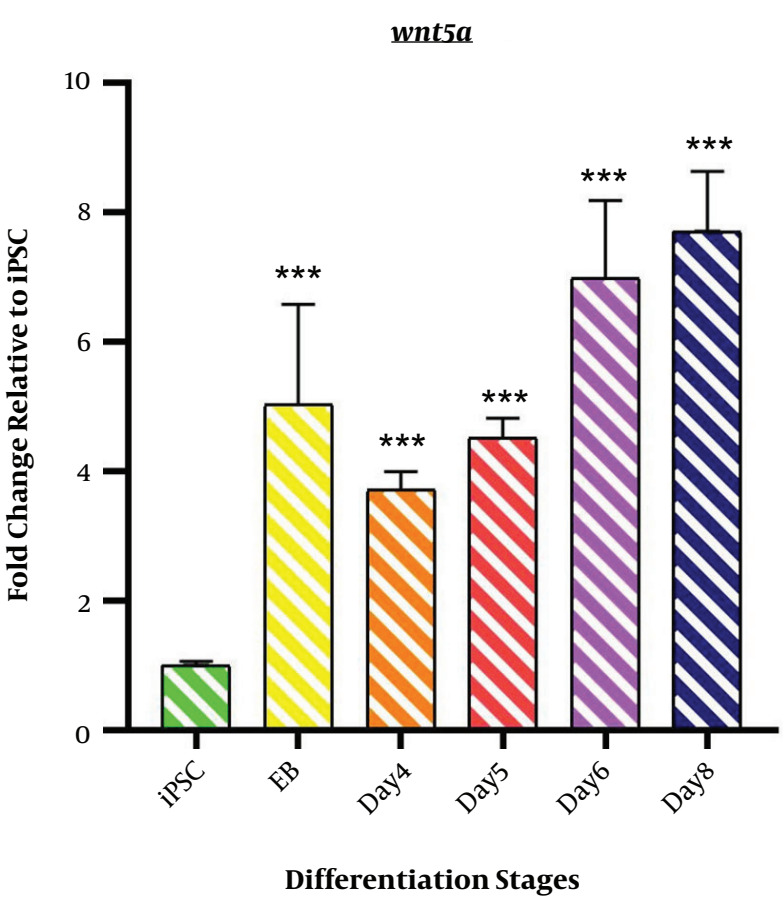

Figure 2. Expression profile of Wnt5a gene; The expression pattern of Wnt5a is presented by the fold change compared to the iPSCs on vertical axis at different stages of differentiation induction on horizontal axis, including iPSCs, Embryoid body (EB), day 4 of differentiation (day 4), day 5 of differentiation (day 5), day 6 of differentiation (day 6), and endoderm (day 8). ${ }^{*} \mathrm{P}<0.05$; ${ }^{* *} \mathrm{P}<0.01$; ${ }^{* * *} \mathrm{P}<0.001$; NS: notsignificant.

\section{Footnotes}

Authors' Contribution: Study concept and design: N. A.; analysis and interpretation of data: Sh. L., and L. K.; drafting the manuscript: Sh. L.; critical revision of the manuscript for important intellectual content: N. A., and S. Gh.; statistical analysis and performing experiments: Sh. L.

Conflict of Interests: The authors declare that they have no conflict of interests.

Funding/Support: None.

\section{References}

1. Puri MC, Nagy A. Concise review: Embryonic stem cells versus induced pluripotent stem cells: The game is on. Stem Cells. 2012;30(1):104. doi: 10.1002/stem.788. [PubMed: 22102565].

2. D'Amour KA, Agulnick AD, Eliazer S, Kelly OG, Kroon E, Baetge EE. Efficient differentiation of human embryonic stem cells to definitive endoderm. Nat Biotechnol. 2005;23(12):1534-41. doi: 10.1038/nbt1163. [PubMed: 16258519].

3. Scheibner K, Bakhti M, Bastidas-Ponce A, Lickert H. Wnt signaling: implications in endoderm development and pancreas organogenesis. Curr Opin Cell Biol. 2019;61:48-55. doi: 10.1016/j.ceb.2019.07.002. [PubMed: 31377680].
4. Ghorbani-Dalini S, Azarpira N, Sangtarash MH, Soleimanpour-Lichaei HR, Yaghobi R, Lorzadeh S, et al. Optimization of activin-A: a breakthrough in differentiation of human induced pluripotent stem cell into definitive endoderm. 3 Biotech. 2020;10(5):215. doi: 10.1007/s13205-020-02215-3. [PubMed: 32355589]. [PubMed Central: PMC7186283].

5. Nusse R, Clevers H. Wnt/beta-Catenin Signaling, Disease, and Emerging Therapeutic Modalities. Cell. 2017;169(6):985-99. doi: 10.1016/j.cell.2017.05.016. [PubMed: 28575679].

6. Komiya Y, Habas R. Wnt signal transduction pathways. Organogenesis. 2008;4(2):68-75. doi: 10.4161/org.4.2.5851. [PubMed: 19279717]. [PubMed Central: PMC2634250].

7. Wiese KE, Nusse R, van Amerongen R. Wnt signalling: conquering complexity. Development. 2018;145(12). doi: 10.1242/dev.165902. [PubMed: 29945986].

8. Lien WH, Fuchs E. Wnt some lose some: transcriptional governance of stem cells by Wnt/beta-catenin signaling. Genes Dev. 2014;28(14):151732. doi: 10.1101/gad.244772.114. [PubMed: 25030692]. [PubMed Central: PMC4102759].

9. Nakamura Y, de Paiva Alves E, Veenstra GJ, Hoppler S. Tissue- and stage-specific Wnt target gene expression is controlled subsequent to beta-catenin recruitment to cis-regulatory modules. Development. 2016;143(11):1914-25. doi: 10.1242/dev.131664. [PubMed: 27068107]. [PubMed Central: PMC4920159].

10. Fan J, Wei Q, Liao J, Zou Y, Song D, Xiong D, et al. Noncanonical Wnt signaling plays an important role in modulating canonical Wntregulated stemness, proliferation and terminal differentiation of hepatic progenitors. Oncotarget. 2017;8(16):27105-19. doi: 10.18632/oncotarget.15637. [PubMed: 28404920]. [PubMed Central: PMC5432321].

11. Clark CE, Nourse CC, Cooper HM. The tangled web of non-canonical Wnt signalling in neural migration. Neurosignals. 2012;20(3):202-20. doi: 10.1159/000332153. [PubMed: 22456117].

12. Gomez-Orte E, Saenz-Narciso B, Moreno S, Cabello J. Multiple functions of the noncanonical Wnt pathway. Trends Genet. 2013;29(9):54553. doi: 10.1016/j.tig.2013.06.003. [PubMed: 23846023].

13. Steinhart Z, Angers S. Wnt signaling in development and tissue homeostasis. Development. 2018;145(11). doi: 10.1242/dev.146589. [PubMed: 29884654].

14. Reya T, Duncan AW, Ailles L, Domen J, Scherer DC, Willert K, et al. A role for Wnt signalling in self-renewal of haematopoietic stem cells. Nature. 2003;423(6938):409-14. doi: 10.1038/nature01593. [PubMed: 12717450].

15. Gessert S, Kuhl M. The multiple phases and faces of wnt signaling during cardiac differentiation and development. Circ Res. 2010;107(2):186-99. doi: 10.1161/CIRCRESAHA.110.221531. [PubMed: 20651295].

16. Keller KC, Ding H, Tieu R, Sparks NR, Ehnes DD, Zur Nieden NI. Wnt5a supports osteogenic lineage decisions in embryonic stem cells. Stem Cells Dev. 2016;25(13):1020-32. doi: 10.1089/scd.2015.0367. [PubMed: 26956615].

17. Nishizuka M, Koyanagi A, Osada S, Imagawa M. Wnt4 and Wnt5a promote adipocyte differentiation. FEBS Lett. 2008;582(21-22):3201-5. doi: 10.1016/j.febslet.2008.08.011. [PubMed: 18708054].

18. Conner DA. Mouse embryo fibroblast (MEF) feeder cell preparation. Curr Protoc Mol Biol. 2000;51(1):23.2.1-7.

19. Choi HK, Yuan H, Fang F, Wei X, Liu L, Li Q, et al. Tsc1 regulates the balance between osteoblast and adipocyte differentiation through autophagy/notch1/beta-catenin cascade. J Bone Miner Res. 2018;33(11):2021-34. doi: 10.1002/jbmr.3530. [PubMed: 29924882]. [PubMed Central: PMC6248888].

20. Baksh D, Boland GM, Tuan RS. Cross-talk between Wnt signaling pathways in human mesenchymal stem cells leads to functional antagonism during osteogenic differentiation. J Cell Biochem. 2007;101(5):1109-24. doi: 10.1002/jcb.21097. [PubMed: 17546602]. 
21. Topol L, Jiang X, Choi H, Garrett-Beal L, Carolan PJ, Yang Y. Wnt-5a inhibits the canonical Wnt pathway by promoting GSK-3-independent beta-catenin degradation. J Cell Biol. 2003;162(5):899-908. doi: 10.1083/jcb.200303158. [PubMed: 12952940]. [PubMed Central: PMC2172823].

22. Sato A, Yamamoto H, Sakane H, Koyama H, Kikuchi A. Wnt5a regulates distinct signalling pathways by binding to Frizzled2. EMBO J. 2010;29(1):41-54. doi: 10.1038/emboj.2009.322. [PubMed: 19910923]. [PubMed Central: PMC2808370].

23. Bengoa-Vergniory N, Gorrono-Etxebarria I, Gonzalez-Salazar I, Kypta RM. A switch from canonical to noncanonical Wnt signaling mediates early differentiation of human neural stem cells. Stem Cells. 2014;32(12):3196-208. doi:10.1002/stem.1807. [PubMed: 25100239].

24. Paige SL, Osugi T, Afanasiev OK, Pabon L, Reinecke H, Murry CE. Endogenous Wnt/beta-catenin signaling is required for cardiac differ- entiation in human embryonic stem cells. PLoS One. 2010;5(6). e11134. doi: 10.1371/journal.pone.0011134. [PubMed: 20559569]. [PubMed Central: PMC2886114].

25. Vijayaragavan K, Szabo E, Bosse M, Ramos-Mejia V, Moon RT, Bhatia M. Noncanonical Wnt signaling orchestrates early developmental events toward hematopoietic cell fate from human embryonic stem cells. Cell Stem Cell. 2009;4(3):248-62. doi: 10.1016/j.stem.2008.12.011. [PubMed: 19265664]. [PubMed Central: PMC2742366].

26. Peng L, Ren LB, Dong G, Wang CL, Xu P, Ye L, et al. Wnt5a promotes differentiation of human dental papilla cells.IntEndod J.2010;43(5):40412. doi: 10.1111/j.1365-2591.2010.01693.x. [PubMed: 20518933].

27. Bradley EW, Drissi MH. WNT5A regulates chondrocyte differentiation through differential use of the CaN/NFAT and IKK/NF-kappaB pathways. Mol Endocrinol. 2010;24(8):1581-93. doi: 10.1210/me.2010-0037. [PubMed: 20573686]. [PubMed Central: PMC5417459]. 